\title{
Kinetics of retroviral production from the amphotropic $\Psi$ CRIP murine producer cell line
}

\author{
Bing Q. Shen ${ }^{1}$, Michael F. Clarke ${ }^{2}$ \& Bernhard O. Palsson ${ }^{1}$ \\ ${ }^{1}$ Departments of Chemical Engineering and ${ }^{2}$ Internal Medicine, The University of Michigan, Ann Arbor, MI \\ 48109, USA
}

Key words: cell culture, half-life, packaging cells, retrovirus, titer, $\Psi$ CRIP

\begin{abstract}
Rapidly expanding development and practice of gene therapy requires the availability of large quantities of high titer retroviral supernatants. One way to achieve high retroviral titers is through improved understanding of the kinetics of retroviral production and decay, and the subsequent development of improved cell culture methods. In the present study we investigated the effects of different operational modes on the retroviral production of the NIH $3 \mathrm{~T} 3$ fibroblast derived amphotropic murine retroviral producing cell line $\mathrm{pMFG/ \Psi CRIP.} \mathrm{Semi-continuous} \mathrm{culture}$ (exchange of $50 \%$ of medium volume daily) was found to promote cell growth and enhance retroviral production. The rapid medium exchange resulted in significantly larger amounts of high titer supernatants and an extended production phase as compared to the batch control cultures. The specific viral productivity of the pMFG/ $\Psi C R I P$ cells was in the range of 10 to 40 infectious viruses produced per thousand producer cells per day. The CV-1 African Green Monkey kidney cell line was used as the infection target. Lowering the serum level from $20 \%$ to $10 \%$ improved retroviral production slightly. However, at lower serum levels $(1 \%, 5 \%$ and $10 \%(\mathrm{v} / \mathrm{v}))$ growth of the producer cell line, and thus retroviral production, was directly proportional to the serum level. The half-life of the virus at $37^{\circ} \mathrm{C}$ was found to be 5.5 hours. Promoting the growth of producer cell lines can improve retroviral vectors titers and viral production. High cell density systems that allow for rapid cell growth and waste product removal are likely to be used to generate high-titer retroviral supernatants.
\end{abstract}

\section{Introduction}

There is presently great interest in the introduction of new genes into primary human cells to enable gene therapy (Verma, 1990). These genetically transformed human cells have a wide potential application in clinical medicine (Bluestone, 1992). The goals are to correct an identified genetic defect or be able to produce a therapeutically beneficial agent in situ. Thus, one of the more promising applications of tissue engineering is the construction of genetically altered human cells for transplantation and thus implementation of gene therapy.

As an alternative to the delivery of doses of pharmacological molecules, the principle underlying gene therapy is to deliver a functional gene whose RNA or protein product will produce the desired biochemical effect in the target cells or tissues. There are several potential advantages of gene therapy over classical biochemical pharmacology. The inserted genes can produce extremely complex molecules, including DNA, RNA, and proteins, which can be extraordinarily difficult to administer and deliver themselves. The controlled insertion of the desired gene into specific target cells can control the production of gene product to defined tissue. Finally, gene therapy can in principle be permanent within an individual, as the gene will continue to function in the target cells and their progeny. The prime example of such a target cell is the human bone marrow stem cell.

Thus, gene therapy has broad application to both inherited and acquired diseases. For example, 
hemoglobin abnormalities such as $\mathbf{a}$ and $\mathbf{b}$ Thalassemias or sickle cell anemia could be treated by the insertion of the gene coding for the globulin a or $b$ or fetal globulin chain, together with regulatory sequences that confer high level tissue-specific expression of these genes, into erythrocytes (Grosveld et al., 1987; Sunshine et al., 1979). In addition to treating inherited enzymatic abnormalities of the hematopoietic system, stem cell gene therapy could be useful for protecting stem cells and their progeny from toxic exogenous agents such as viruses or chemotherapy. For example, gene transfer of DNA sequences encoding the TAR binding site of the HIV TAT transactivating factor have been shown to protect $\mathrm{T}$ cells from speading infection by the HIV virus (Sullenger et al., 1990). Stable transfection of these sequences into hematopoietic stem cells would result in a pool of $\mathrm{T}$ cells, all arising from the stem cells, which were relatively or absolutely resistant to the spread of HIV.

There are several problems that must be addressed and dealt with successfully to enable gene therapy. The first issue is to be able to insert the desired therapeutic gene into the chosen cells. Second, the gene must be adequately expressed in the target cells, resulting in the appropriate levels of gene product. Finally the DNA, RNA, or protein produced must be properly processed by the target cells so that it is functional, i.e. so that gene therapy actually infers clinical therapy. To accomplish these goals, several methods of gene insertion into human cells in vitro have been developed. Presently, the major emphasis in gene therapy utilizes a replication-defective retrovirus as the delivery system (Miller, 1990).

Unlike most other viruses which eventually cause lysis of recipient cells, retroviruses infect host cells and integrate into the host chromosomes in the form of proviruses (Butler, 1991; Larrick and Burck, 1991). An infectious replication-defective recombinant retroviral vector is designed to have cis functions including the long terminal repeats (LTRs) - sequences for the promoter and enhancer, the packaging signal and the tRNA primer binding site. Three trans genes, gag, pol and env, are provided by the packaging cell line. In general, the therapeutic gene replaces the structural gene in the therapeutic vector.

The first step in the infection process - the binding of the virion to the target cell - is limited by the amount of retrovirus present in the supernatant used for infection. Thus high retroviral titers are needed to improve infectivity. One way to improve the titers obtained from the producer cell lines is to properly design the culture conditions. To begin to understand the culture parameters which control the amount of virus produced and secreted, we have measured the kinetics of viral production of an amphotropic retroviral producer $\mathrm{pMFG} / \Psi \mathrm{CRIP}$ and the degradation of the retrovirus produced. The results are reported herein.

\section{Materials and methods}

\section{Cell lines and culture medium}

The amphotropic producer cell line pMFG/ $\Psi$ CRIP was kindly provided by Dr. James Wilson at The University of Michigan. The packaging cell line $\Psi$ CRIP was derived from fibroblast NIH 3T3 and originally established by Danos and Mulligan (Danos and Mulligan, 1988). It has two recombinant vectors pCRIPenv ${ }^{-}$, encoding for gag and pol, and pCRIPAMgag-, encoding for 4070A amphotropic virus genome. This packaging cell line was further transduced with plasmid pMFG, consisting of LTRs, packaging signal $Y$ (psi) and lacZ, and formed the amphotropic retroviral producer cell line $\mathrm{pMFG} / \Psi \mathrm{CRIP}$. The marker gene lacZ encodes bacterial $\beta$-galactosidase, which can be easily used to stain positively infected target cell colonies. These cells were grown in Dulbecco's Modified Eagle's medium (DMEM) (GIBCO, cat. no. 4302800EB) supplemented with $10 \%$ fetal bovine serum (FBS) (GIBCO, cat. no 240-6000AJ). Various levels of serum were used for the study of retroviral production.

$\mathrm{CV}-1$ cells were used to titrate the virus supernatants. They are fibroblast-like cells from African Green Monkey (Cercopithecus aethiops) kidney (ATCC CCL 70). They were grown in DMEM supplemented with $10 \%(\mathrm{v} / \mathrm{v})$ fetal bovine serum or calf serum.

Both pMFG/ $\Psi C R I P$ and $C V-1$ cells are anchoragedependent cell lines. In both cases the cell passage procedure involved three steps; first, rinsing with $\mathrm{Ca}^{+2}$ and $\mathrm{Mg}^{+2}$-free Hank's Balanced Salt Solution (HBSS) (GIBCO, cat. no. 310-4180AJ); second, treatment with trypsin-EDTA (GIBCO, cat. no. 610-5400PE) and; third, neutralization with $10 \%$ serum DMEM. Cell suspensions then were split at a ratio of 1 to 10 and replated. 


\section{Cultivation of pMFG/ $\Psi$ CRIP and production of retroviral supernatants}

Three cultivation methods were investigated for the

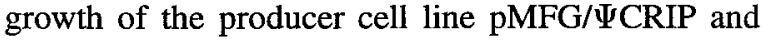
retroviral production:

Batch culture with conditioning $(B C W C)$. In this commonly used approach, the producer cells were allowed to grow reaching $50 \%$ to $70 \%$ of confluence, and then the medium was totally replaced by fresh medium. Viral supernatants were collected after conditioning medium with the producer cells for 12 to 24 hours. We studied cell growth and viral production under this scenario on a daily basis. Ten six-well (Costar, 3406) plates were inoculated with $5 \times 10^{4}$ pMFG/ $\Psi C R I P$ cells per well in $3 \mathrm{ml}$ of $10 \%$ FBS DMEN. On each day following, one plate was used to determine cell growth and to produce retrovirus for 19 hours in fresh $10 \%$ and $20 \%$ FBS DMEM. Cells in two wells (duplicates) were trypsinized, removed, and counted to measure cell growth. Three milliliters of $10 \%$ and $20 \%$ FBS SMEM were added to each of the four remaining wells (duplicates) and viral supernatants were collected after about 19 hours.

Batch culture $(B C)$. Under this scenario, there was no medium replacement after inoculation $1.2 \times 10^{5}$ pMFG/ $\Psi$ CRIP cells were seeded in ten $85 \mathrm{~mm}$ tissue culture dishes in $10 \mathrm{ml}$ of $10 \%$ CSS DMEM. One dish was taken down for cell counting, titration of retrovirus produced, and analysis of glucose, glutamine, and ammonium concentrations in the medium.

Semi-continuous culture (SCC). After the producer cells were inoculated, a daily partial or compiete replacement of medium was carried out over the period of time of incubation. Ten $85 \mathrm{~mm}$ tissue culture dishes were seeded with $1.2 \times 10^{5}$ pMFG/ $\Psi$ CRIP cells per plate in $10 \mathrm{ml}$ of $10 \%$ CSS DMEM. One plate was sacrificed daily for cell counting, viral titration, and analysis of substrates and metabolic byproducts; the rest of the dishes received a daily medium replacement of $5 \mathrm{ml}$ from day 1 to day 4 but $50 \%$ from day 5 to day 9.

The effects of serum level were compared in a separate experiment where the growth medium contained $1 \%, 5 \%$ and $10 \%$ CSS.

All virus preparations were filtered before use through $0.45 \mathrm{~mm}$ filters (Gelman Sciences, product no. 4184 ) to remove any free producer cells in the super- natants and prevent in situ transfection of CV-1 cells. Prior to use the filters were pretreated with appropriate serum-containing media $(10 \%, \mathrm{v} / \mathrm{v})$.

\section{Titration of virus}

Twenty to twenty-five thousand CV-1 cells were inoculated to each well of a six-well plate (Costar, 3406) with $3 \mathrm{ml}$ of growth media and incubated for 12 to 18 hours before the introduction of retrovirus. After removing the spent medium, $0.2 \mathrm{ml}$ of virus solution was added into a well, plus $1.8 \mathrm{ml}$ growth medium containing $4.44 \mu \mathrm{g} / \mathrm{ml}$ of polybrene (Aldrich, cat. no. $10,768-9$ ). After 8 to 10 hours at $37^{\circ} \mathrm{C}$, the infection medium was replaced by $3 \mathrm{ml}$ of growth medium to support growth of $\mathrm{CV}-1$ cells to confluence. Having been washed twice by $\mathrm{Ca}^{+2}$ and $\mathrm{Mg}^{+2}$-free Dulbecco's phosphatebuffered saline (GIBCO, cat. no. 310-4080AJ), the confluent infected $\mathrm{CV}-1$ cells in each well were fixed by $1.5 \mathrm{ml}$ of $2.1 \%(\mathrm{v} / \mathrm{v})$ formaldehyde (Sigma, cat. no. F-1635) and $0.2 \%(\mathrm{v} / \mathrm{v})$ glutaraldehyde (Sigma, cat. no. G-6257) for five minutes. The cells were washed with PBS twice again. A staining solution was prepared by adding $50 \mathrm{ml}$ of $20 \mathrm{mg} / \mathrm{ml}(2 \%)$ X-gal (5-Bromo-4chloro-3-indolyl- $\beta$-D-galactoside) (GIBCO BRL, cat. no. 5520UC) dissolved in N,N-Dimethylformamide (DMF) (Sigma, cat. no. D-8654) into each milimiter of the solution containing $5 \mathrm{mM} \mathrm{K}_{3} \mathrm{Fe}(\mathrm{CN})_{6}$ (Sigma, cat no. P-3667), $5 \mathrm{mM} \mathrm{K}_{4} \mathrm{Fe}(\mathrm{CN})_{6} \cdot 3 \mathrm{H}_{2} \mathrm{O}$ (Sigma, cat. no. P-3289) and $2 \mathrm{mM} \mathrm{MgCl}$ (Sigma, cat. no. M-8266) immediately before use. To each well, $1.5 \mathrm{ml}$ of staining solution was added and the plates were put into a $37^{\circ} \mathrm{C}$ incubator for 8 to 12 hours. The stained positive (blue or green) colonies were counted and the titer of the retroviral solution was calculated as the number of colony formation units per milliliter of virus solution used (CFU/ml).

To make sure that the infections were carried out under conditions where titers were not underestimated because the target cells were superinfected, a dosage response experiment was carried out. These results show a clear linear relationship between the colony formation unit (CFU) reading and the volume of virus supernatant used (Figure 1a). Thus, the infection assay was not performed under conditions where superinfections occured.

To compare the infection efficienty of the CV-1 cells to the commonly used NIH 3 T 3 cells, we titrated the same viral supernatants on CV-1 and NIH 3 T3 cells in parallel. The results showed that the NIH $3 \mathrm{~T} 3$ murine 

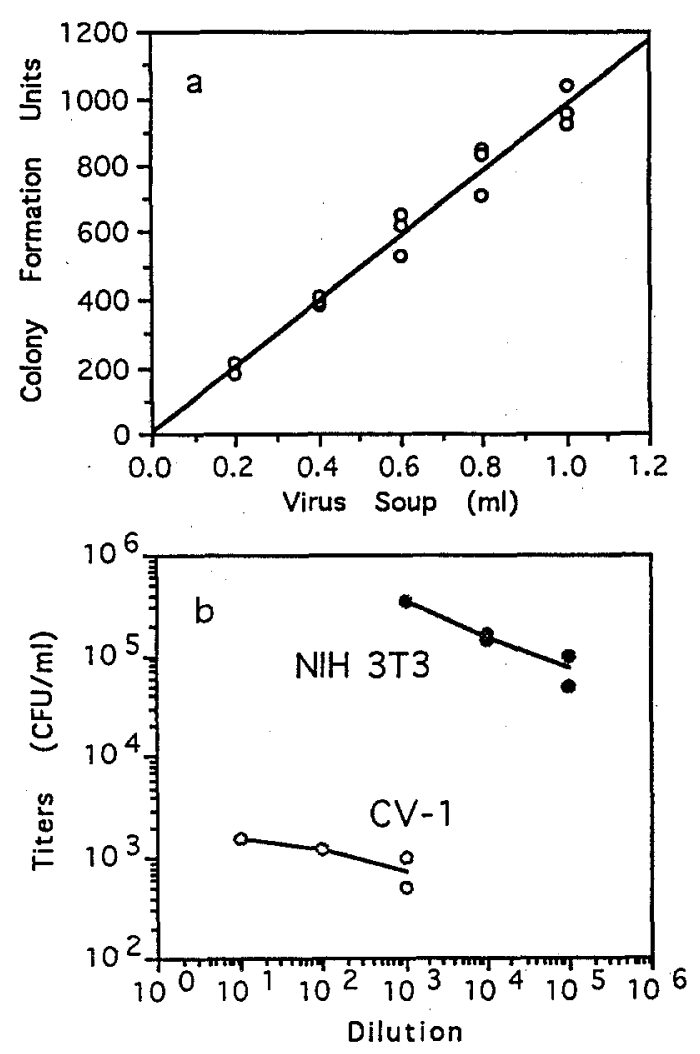

Figure 1. (a) The dosage response of colony formation unit (CFU) reading. (b) The titer responses of CV-1 and NIH 3T3 cells to a retroviral supernatant, produced by $\mathrm{pMFG} / \Psi \mathrm{CRIP}$, at different dilutions. $0.2 \mathrm{ml}$ of serially diluted retroviral solutions were added to each well of six-well plates which were inoculated with CV-1 and NIH 3T3, respectively, at the level of 25,000 cells per well 10 hours before infection. Then $1.8 \mathrm{ml}$ of $10 \%$ CSS DMEM containing $4.44 \% \mathrm{mg} / \mathrm{ml}$ of Polybrene was added to each well. The infection was carried out for 8 hours in an incubator and then the infection medium was replaced with fresh medium to support the cells growing until confluence and stained with X-Gal.

cells have infectivities that are about 300 times higher than CV-1. (Figure 1b).

\section{Analysis of glucose, lactate, glutamine, and ammonium}

Glucose and lactate in medium samples were measured with a glucose/lactate analyzer (Yellow Springs Instruments, Model 2000, Yellow Springs, $\mathrm{OH}$ ). Glutamine and ammonium concentrations were analyzed with a L-glutamic acid kit (Boehringer Mannheim, 139092) in conjunction with L-asparaginase (Boehringer
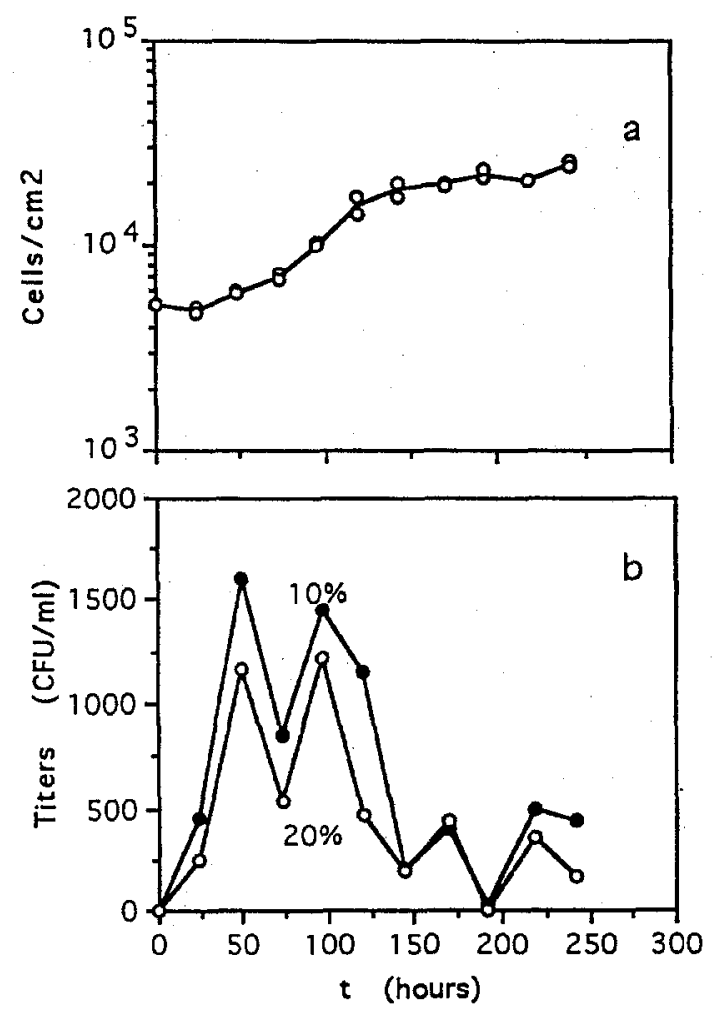

Figure 2. The growth of producer cell pMFG/ $\Psi$ CRIP (a) and production of retrovirus (b) in batch culture with conditioning (10\% and 20\% FBS DMEM).

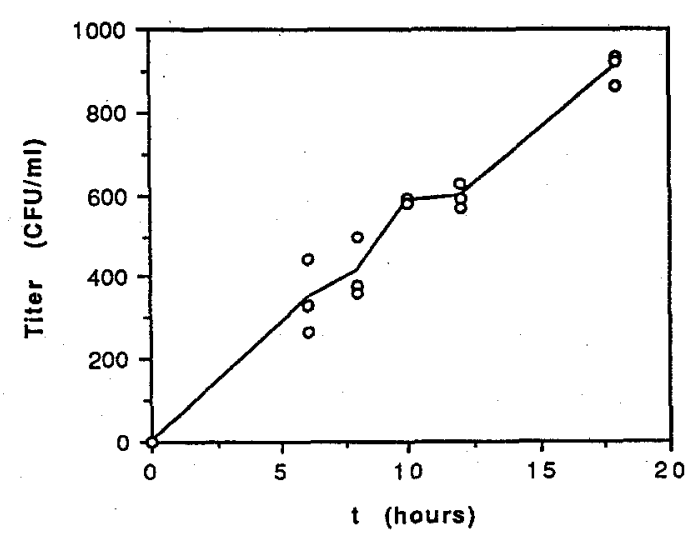

Figure 3. Production kinetics of retrovirus in DMEM with $10 \%$ FCS. The concentration profile of retrovirus produced during conditioning with $10 \%$ FBS DMEM.

Mannheim, 102903), and an ammonia kit (Boehringer Mannheim, 1112732), respectively. 

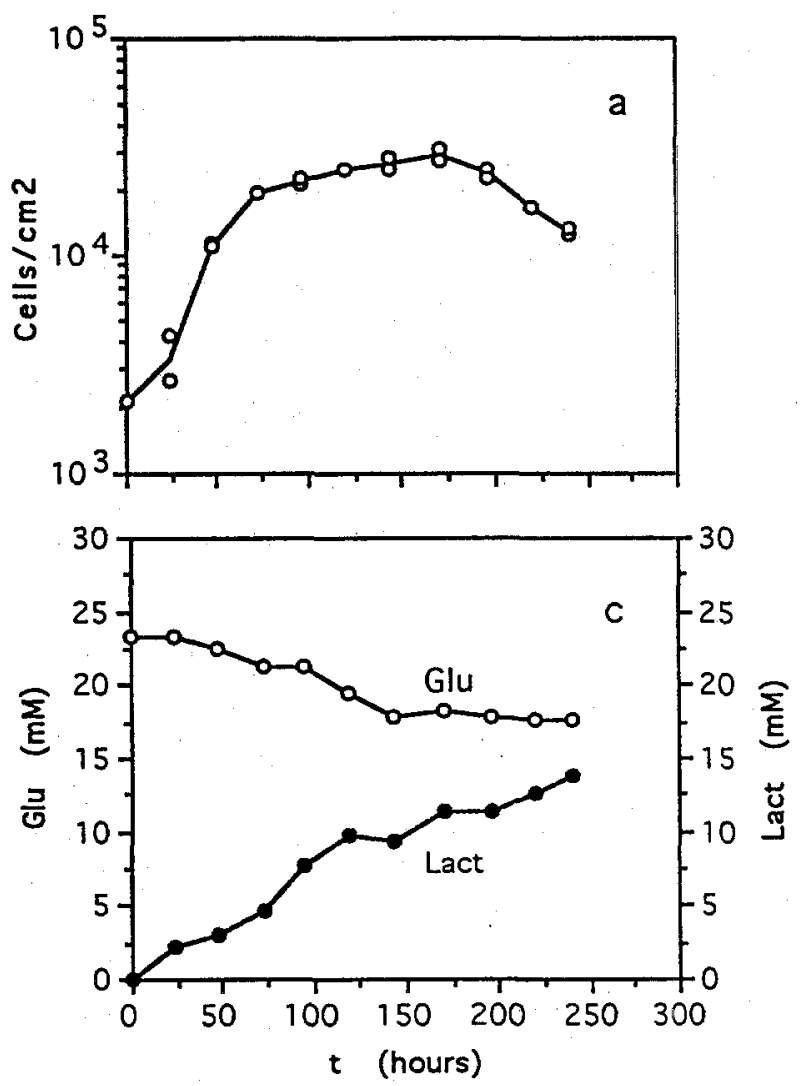
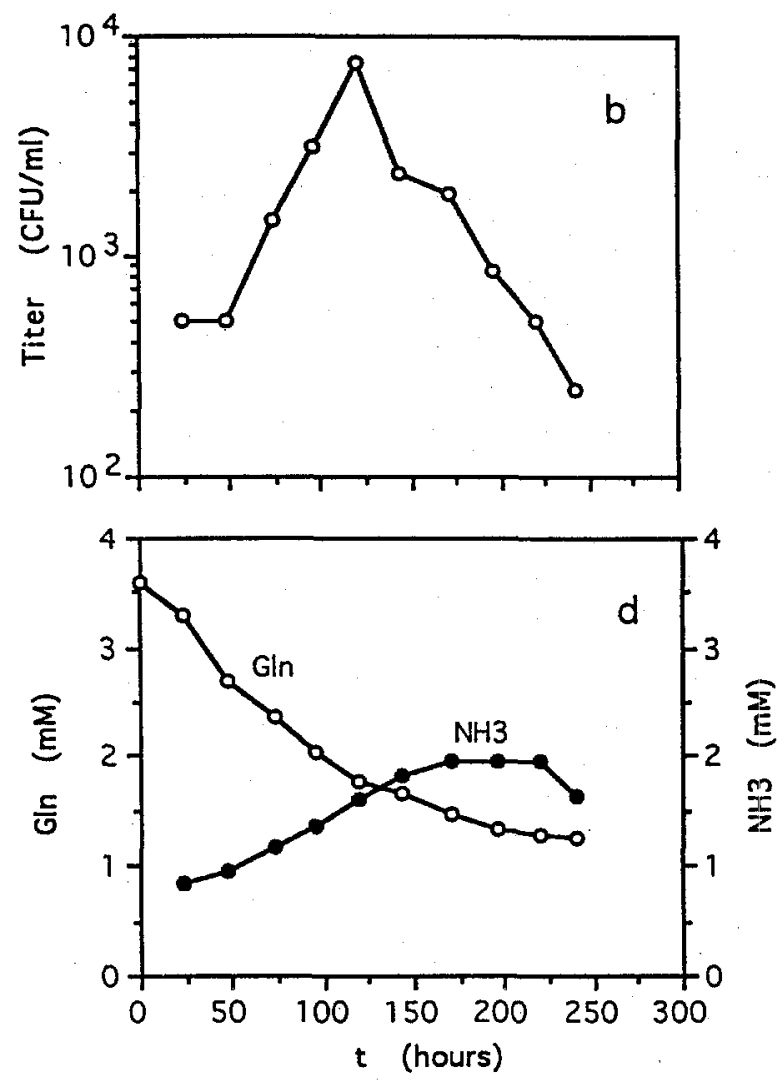

Figure 4. Batch culture of producer cell pMFG/ $\Psi$ CRIP and its retroviral production (a), and concentration profiles of glucose-lactate (b) and glutamine-ammonia (c).

\section{Results}

\section{Retroviral production in a batch culture with conditioning (BCWC)}

The pMFG/ $\Psi$ CRIP cells grew to a maximum cell density of about $2.1 \times 10^{4}$ cells $/ \mathrm{cm}^{2}$ following a short lag phase (Figure 2a). Unlike the parental cell line, these cells continued to grow after a confluent monolayer had been formed. Multiple cell layers were observed following day six of cultivation. Viral production was measured by a complete medium replacement at a particular point in time and the concentration of virus was allowed to build up for 18 hours in the fresh medi$u m$. The viral titer increased in a linear fashion during the 18 hour period (Figure 3 ) and the cell population typically doubled during the viral production period.

Viral production was active between day 2 and day 5 , but began to decline when subconfluence was reached (Figure $2 b$ ). The production of retrovirus in the presence of $20 \%$ FBS was consistently lower than that in $10 \%$ FBS medium. The retroviral titers normally achieved in BCWC mode were around $10^{3} \mathrm{CFU} / \mathrm{ml}$, using CV-1 cells as the infection target.

\section{Retroviral production in batch culture $(B C)$}

In batch culture (BC), retroviral producer pMFG/ ICRIP cells exhibited a three-day period of rapid growth with a maximal growth rate of about $5.0 \times$ $10^{-2} \mathrm{hr}^{-1}$. Then cell growth slowed and the culture reached a maximum cell density of about $2.8 \times 10^{4}$ cells $/ \mathrm{cm}^{2}$ on the seventh day (Figure $4 \mathrm{a}$ ). The viral titer increased dramatically while the cells were growing rapidly and reached maximum level of $7.5 \times 10^{3}$ $\mathrm{CFU} / \mathrm{ml}$ on the fifth day. The titer increased while the cells were in stationary phase but declined dramatically following loss of viability.

It should be noted that the peak in the retroviral titer was not always observed. Our highest titer in BC 

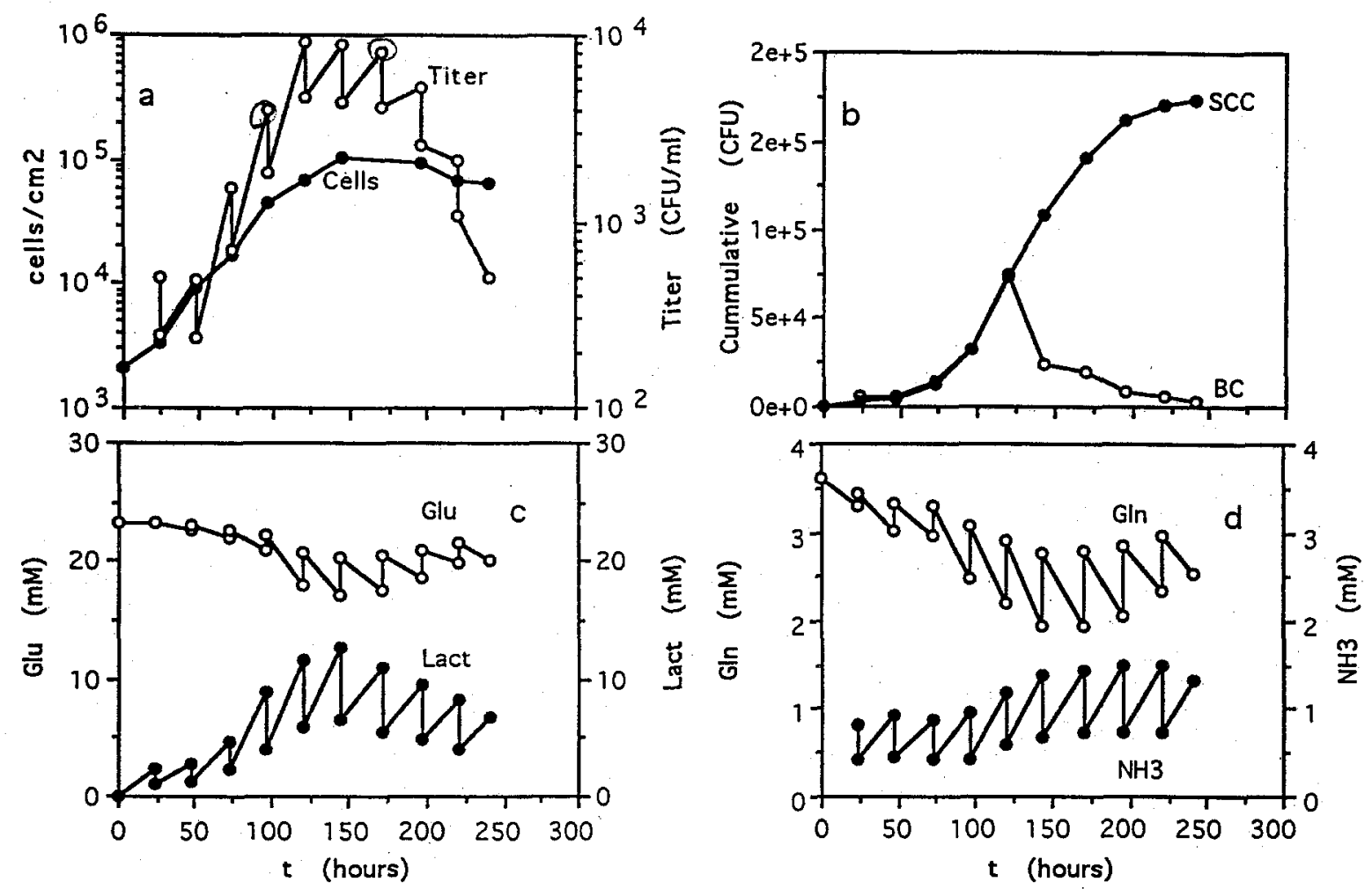

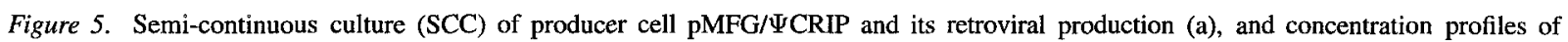
glucose-lactate (b) and glutamine-ammonia (c).

is shown in Figure 4a. Thus, there is a peak point in the titer curve that is quite sharp and whose timing is difficult to predict. Thus, production of virus of $\mathrm{BC}$ to obtain a high titer is unreliable, especially if large quantities of high titer supernatants are needed.

Analysis of glucose and glutamine concentrations showed that they were the main substrates for cell growth (Figure $4 \mathrm{~b}$ and $4 \mathrm{c}$ ). Neither of those limited growth; their concentrations were $17.6 \mathrm{mM}$ of glucose and $1.24 \mathrm{mM}$ of glutamine, at the end of batch culture. Interestingly, both - though glutamine more significantly - were consumed after cessation of growth (at approximately 75 hours). The major metabolic byproducts, lactate and ammonium, reached $13.8 \mathrm{mM}$ and $2 \mathrm{mM}$, respectively.

\section{Retroviral production in semi-continuous culture (SCC)}

To promote cell growth and viral production, a rapid medium exchange protocol was used where about one half of the medium was exchanged on a daily basis. Over the first six days of culture the cell density increased exponentially at a maximal apparent specific growth rate of $4.4 \times 10^{-2} \mathrm{hr}^{-1}$ that is slightly lower than obtained in BC. The cell density reached a maximum of $1.04 \times 10^{5} \mathrm{cells} / \mathrm{cm}^{2}$ (Figure 5a), approximately a four-fold higher cell density than in BC. Multiple cell layers were observed.

Neither of the major nutrients was depleted (Figure $5 \mathrm{c}$ and $5 \mathrm{~d}$ ). Furthermore, the SCC leads to lower concentrations of metabolic waste products. Thus, growth in SCC may be limited by the cell growth surface area.

The titer of virus produced exceeded $10^{3} \mathrm{CFU} / \mathrm{ml}$ on day three and was maintained at a higher level of $8 \times 10^{3}$ to $10^{3}$ to $10^{4} \mathrm{CFU} / \mathrm{ml}$ from day 5 to day 7 . Retroviral production was maintained during stationary phase and most of the high titer supernatants are obtained during and shortly after entry into stationary phase. The cumulative colony formation units (CFU) produced in CSS is thus quite high compared to BC, 

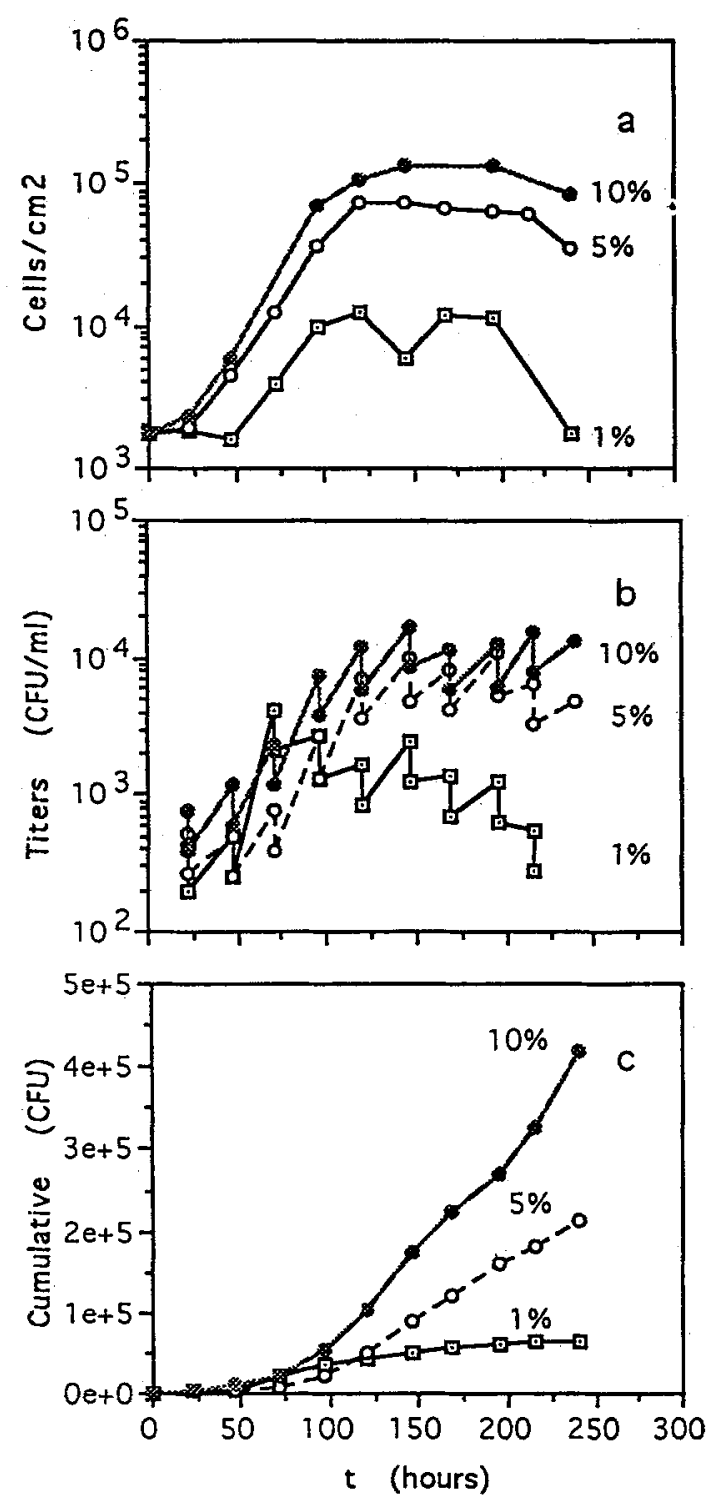

Figure 6. The effect of calf serum concentration on the growth of producer cell pMFG/ $\Psi$ CRIP (a), retroviral productions (b) and cumulative retroviral production (c) in semi-continuous culture.

Figure $5 \mathrm{~b}$. Further, most of this virus obtained at higher concentrations.

Effects of serum concentration on cell growth and retroviral production

To help identify limiting factor(s) in the medium, growth of pMFG/ $\Psi$ CRIP cells and their retroviral pro- duction were studied under the prolific SCC conditions of $50 \%$ daily medium replacement at different serum concentrations $(1 \%, 5 \%$ and $10 \%)$. The dependence of cell growth on the serum in the medium was strong, Figure 6a. The cultures receiving $1 \%, 5 \%$ and $10 \%$ serum reached about $1.4 \times 10^{4}, 6.9 \times 10^{4}$ and 1.4 $\times 10^{5}$ cells per square centimeter respectively. Thus, the final cell number obtained was proportional to the amount of serum used. The use of substrates and formation by byproducts also showed a similar proportion (Figure 7).

Serum thus clearly contains mitrogenic stimulus for the pMFG/ $\Psi$ CRIP cell line. The serum-contained factor that is responsible for the mitrogen stimulus is not known. However, it is known that Platelet Derived Growth Factor (PDGF) and Epidermal Growth Factor (EGF) support the growth of NIH $3 \mathrm{~T} 3$ fibroblasts and they are thus likely candidates (Freshney et al 1987; Reed, 1991). Although the cell yield is proportional to the amount of serum the cells were exposed to, pMFG/ICRIP cells exhibited quite similar maximal growth rate at all serum concentrations, Figure 6a.

As the result of better cell growth, the cultures with higher serum levels showed better viral production, Figure $6 \mathrm{~b}$ and $6 \mathrm{c}$. The titers obtained from the $1 \%$, $5 \%$ and $10 \%$ serum-containing cultures were approximately proportional to the serum concentration. This observation suggests that the viral production is strongly promoted by cell growth. Figure 7 shows the concentration profiles of glucose, lactate, glutamine, and ammonia during the culture of $\mathrm{pMFG} / \Psi \mathrm{CRIP}$ cells in DMEM with different levels of calf serum supplement. The correlation of glucose and glutamine consumption and lactate and ammonium production are presented in Figure 8. The lactate/glocose and ammonium/glutamine yields are essentially constant in both SCC and BC.

\section{Deactivation of retrovirus}

The stability of the retrovirus is one of the most important factors in the development of an optimal strategy for retroviral production and design of infection system. Retroviral sypernatants collected from the culture $0.45 \mathrm{~mm}$ syringe filters and then titrated at certain time intervals. The viral supernatants were put into an incubator at $37^{\circ} \mathrm{C}$ during the course of deactivation. The decay of the retrovirus produced by $\mathrm{pMFG/ \Psi CRIP}$ at $37^{\circ} \mathrm{C}$ is shown in Figure 9. The half-life of the virus estimated from this data is 5.5 hours. 

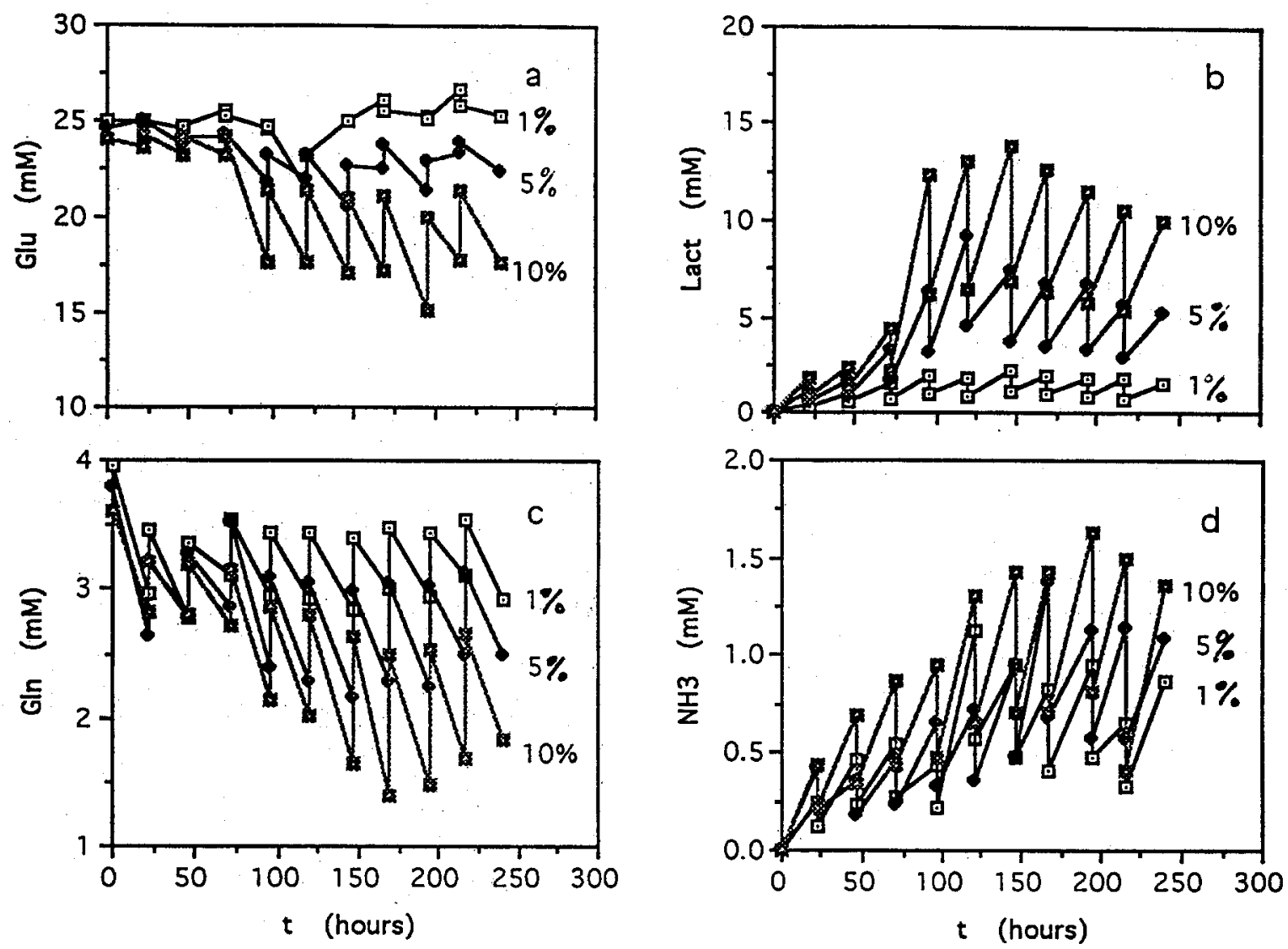

Figure 7. Concentration profiles of glucose (a), lactate (b), glutamine (c) and ammonia (d) during the semi-continuous culture of pMFG/ $\Psi C R I P$ cells in DMEM with $1 \%, 5 \%$ and $10 \%$ calf serum supplement.
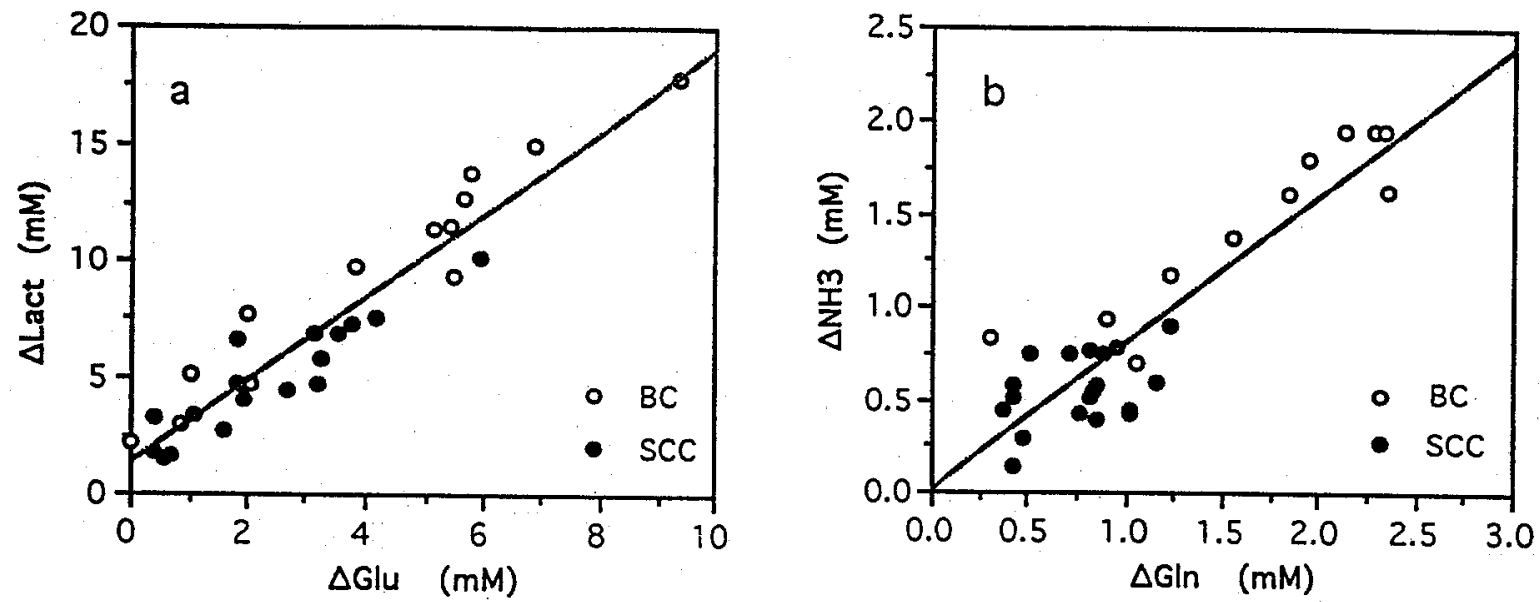

Figure 8. Correlation between glucose consumption and lactate formation (a), glutamine consumption and ammonia formation (b). These data are from the batch $(\mathrm{BC})$ and semi-continuous culture (SCC) of pMFG/ $\Psi$ CRIP cells in $10 \%$ calf serum supplemented DMEM. 


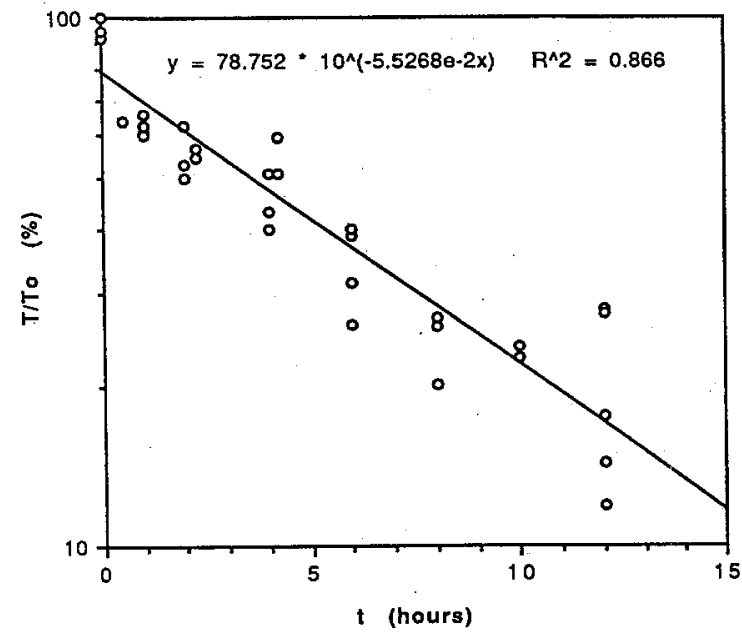

Figure 9. Stability profile of $\mathrm{pMFG} / \Psi \mathrm{CRIP}$ derived retrovirus at $37^{\circ} \mathrm{C}$.

\section{Specific retroviral productivities}

The specific (per cell) retroviral productivity of the pMFG/ $\Psi C R I P$ cell line is readily calculated from the growth curve and the retroviral titer as

$$
\mathrm{q}_{\mathrm{v}}=\mathrm{DC}_{\mathrm{vt}} \mathrm{V}_{\mathrm{t}} / \mathrm{S} \mathrm{X} \mathrm{X}_{\mathrm{vt}} \Delta \mathrm{t}
$$

where $\mathrm{q}_{\mathrm{v}}$ is the specific retroviral productivity (CFU per $10^{3}$ cells per hour), $\mathrm{C}_{\mathrm{vt}}$ is the concentration of virus, $X_{v t}$ is the average viable cell density per unit area over the period of time $(\Delta t)$ of retroviral production, $S / V_{t}$ is the specific surface area. The calculated results for $\mathrm{q}_{\mathrm{v}}$ are presented in Table 1. Based on these data, we estimated that the specific viral productivity during growth and early stationary phase is in the range $10-40 \mathrm{CFU} / 10^{3}$ cells/day, when titrated on CV- 1 cells. The number of NIH 3T3 infective particles is expected to be 300 times higher. The data presented here is too fragmented to correlate $\mathrm{q}_{\mathrm{v}}$ with specific cell parameters, which is likely caused by the relatively high instability of the viral particles.

\section{Discussion}

As detailed in the Introduction, the effective transfer of therapeutic genes into the target cells relies on the availability of retroviral vector preparations of sufficiently high titers. We have studied the kinetics of the retroviral vector producer cell line $\mathrm{pMFG/ \Psi CRIP}$ in order to find an efficient way to produce high titer retroviral supernatants in large quantities. Three operational modes, i.e., batch culture with conditioning (BCWC), batch culture (BC), as well as semi-continuous culture (SCC), were investigated. Based on this study we can draw several important conclusions about the growth, metabolism, and viral production of the $\mathrm{pMFG/ \Psi CRIP}$ producer cell line.

The primary conclusion from the present results is that the production of retrovirus from the pMFG/ $\Psi$ CRIP cell line can be increased by promoting cell growth and cell density. In the batch culture, the accumulation of viral titer followed cell growth closely and reached its maximum before cell viability started to decline (Figure 4). The prolonged high-titer production phase observed in the semi-continuous process (Figures 5 and 6) was evidently due to the extended growth phase of the producer cells. For example, pMFG/ $\Psi$ CRIP cells had an exponential phase of about three days in the batch culture, while in the semicontinuous culture in 10\% CSS DMEM, the cells experienced a six-day active growth phase. This result supports the hypothesis that high cell density bioreactor systems would give high viral titer supernatants.

A semi-continuous perfusion strategy was proven to be effective to improve the growth of producing cell line $\mathrm{pMFG/ \Psi CRIP}$ as well as retroviral production. SCC mode provided an additional supply of growth factors and nutrients which probably were limiting or not sufficient for cell growth and function, and helped to remove metabolic products and other inhibitory compounds such as lactate and ammonium ions. Compared with what was achieved in batch culture, the cell density in SCC mode was about 4 times as high as that of BC mode, indicating a better capability to support cell growth. Although NIH 3T3 cells are reported to be inhibited by ammonium ions (Visek et al., 1992), preliminary data from our laboratory indicates that at the ammonium concentration experienced here growth

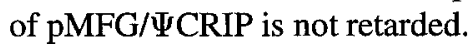

The dependence of pMFG/ $\Psi$ CRIP cell growth and retroviral production on serum was evident in $85 \mathrm{~mm}$ tissue culture dishes, when different concentrations of serum $(1 \%, 5 \%$ and $10 \%)$ were used for medium replacement. The effective components of this limitation are considered most likely to be PDGF and/or EGF, which are considered to be necessary for the growth of NIH 3T3 cells. However, as demonstrated in the BCWC mode, 20\% FBS DMEM consistently gave no boost of viral production. This suggests the existence of agents in serum which inhibit or neutralize the 
Table 1. Specific retroviral productivities calculated in different processes (CFU $/ 10^{3} \mathrm{pMFG} / \Psi \mathrm{CRIP}$ cells/day). The virus was titrated on $\mathrm{CV}-1$ cells

\begin{tabular}{|c|c|c|c|c|c|c|c|}
\hline \multirow{2}{*}{$\begin{array}{l}\text { Time } \\
\text { (days) }\end{array}$} & \multicolumn{7}{|c|}{ Process $^{\mathrm{a}}$} \\
\hline & 1 & 2 & 3 & 4 & 5 & 6 & 7 \\
\hline 1 & 33.3 & 18.3 & 32.0 & 31.7 & 35.1 & 53.4 & 71.1 \\
\hline 2 & 96.7 & 70.7 & 0.2 & 7.8 & 29.0 & 12.3 & 32.3 \\
\hline 3 & 37.9 & 23.7 & 10.2 & 15.8 & 225 & 9.9 & 21.4 \\
\hline 4 & 43.8 & 36.7 & 14.2 & 18.1 & 12.4 & 14.1 & 21.3 \\
\hline 5 & 26.7 & 10.9 & 29.9 & 21.1 & 5.0 & 15.7 & 13.9 \\
\hline 6 & 4.2 & 4.0 & & 7.5 & 24.1 & 11.4 & 12.7 \\
\hline 7 & 7.5 & 8.1 & & 6.0 & 3.0 & 7.9 & 3.8 \\
\hline 8 & 0.6 & 0.1 & & 1.2 & 6.0 & 13.3 & 6.4 \\
\hline 9 & 8.6 & 6.2 & & & & 2.7 & 12.6 \\
\hline 10 & 7.0 & 2.6 & & & & 4.84 & 7.8 \\
\hline
\end{tabular}

a 1 - BCWC, $10 \%$ FBS;

2-BCWC, $20 \%$ FBS;

$3-\mathrm{BC}, 10 \%$ CSS;

$4-\mathrm{SCC}, 10 \%$ CSS;

5 - SCC, $1 \%$ CSS;

$6-\mathrm{SCC}, 5 \% \mathrm{CSS}$

7 - SCC, $10 \%$ CSS.

viruses produced resulting in lower titer supernatants (Aaronson and Stephenson, 1974; Levy et al., 1975; Stephenson et al., 1976).

Viral productivity and titers can be enhanced by improving growth conditions. Viral production in both growth and stationary phase have been observed, and undoubtedly ceases as cell viability decreases. The production of infectious retrovirus particles by the producer cell line pMFG/YCRIP depends on the appropriate expression and assembling of their products of the genes gag (viral core protein), pol (reverse transcriptase) and env (viral envelope) into viral particles, and most importantly, the inclusion of viral RNA into the viral particles. Because these viral genes are encoded on three separately located DNA constructs, the formation of viral elements may not be in the right proportion as required by the composition of viral structure. One element may be the limiting factor for the assembly of infectious viral particles. How the cell cycle status of the producer cells affect the expression of these genes is not known. Among the virus particles produced, some may have no viral RNA at all (Butler, 1991). Some may have no, or improperly, translated env products and are therefore not infectious (Fields et al., 1990).

The per cell productivity was found to be approximately a constant, at 10 to $40 \mathrm{CV}-1$ infectious viral particles per thousand producing cells per day (Table 1). However, as shown in Figure 1b, the susceptibility of NIH 3T3 cells is 300 times higher than that of CV-1 cells to the viruses produced by pMFG/ICRIP. The reason for this difference is unknown. Both growth and stationary phase should be suitable for use in high density systems. The virus would be secreted into a relatively small culture volume leading to high titers. Although batch and semi-continuous culture modes are useful ways to produce retrovirus from producer cell lines that have growth-related viral production, an operational strategy can also be developed by extending cell growth in high cell density systems, thus prolonging the viral production phase.

\section{Acknowledgements}

We would like to thank Dr. J. M. Wilson for providing the producer cell line, and Aastrom Biosciences, Inc. for funding this work.

\section{References}

Aaronson SA and Stephenson JR (1974). Widespread natural occurrence of high titers of neutralizing antibodies to a specific class of endogenous mouse type-C virus. Proc Nat Acad Sci USA 71(5): 1957-1861.

Bluestone M (1992). Genes in a bottle. Bio/technol 10: 132-136. Butler M (1991), Mammalian Cell Biotechnology. IRL Press. 
Danos O and Mulligan RC (1988). Safe and efficient generation of recombinant retroviruses with amphotropic and ecotropic host ranges. Proc Nat Acad Sci USA 85: 6460-6464.

Fields BN, Knipe DM, Chanock RM, Hirsch MS, Melnick JL and Monath TP (1990). Virology (2nd ed.), volume 1. Raven Press, Ltd., New York.

Freshney RI (1987), Culture of animal cells - a manual of basic technique. 2nd edition. Wiley-Liss.

Grosveld F, van Assendelft GB, Greaves DR and Kollias G (1987). Position-dependent, high-level expression of the human betaglobin gene in transgenic mice. Cell 51(7): 975-985.

Larrick JW and Burck KL (1991). Gene Therapy - Application of molecular biology. Elsevier.

Levy JA, Ihle JN, Oleszko O and Barnes RD (1975). Virus-specific neutralization by a soluble non-immunoglobulin factor found naturally in normal mouse sera. Proc Nat Acad Sci USA 72(12): $5071-5075$.

Miller AD (1990). Retrovirus packaging cells. Human Gene Therapy 1: 5-14.

Reed JC, Talwar HS, Cuddy M, Baffy G, Williamson J, Rapp UR and Fisher GJ (1991). Mitochondrial protein p26 BCL2 reduces growth factor requirements of NIH3T3 fibroblasts. Exp Cell Res 195: 277-283.

Stephenson JR, Peters RL, Hino S, Donahoe RM, Long LK, Aaronson SA and Kelloff GJ (1976). Natural immunity in mice to structural polypeptides of endogenous type-C RNA viruses. J Virol 19(2): 890-898.

Sullenger BA, Gallardo HF, Ungers GE and Gilboa E (1990). Overexpression of TAR sequences renders cells resistant to human immunodeficiency virus replication. Cell 63(3)L 601-608.

Sunshine HR, Hofricher J and Eaton WA (1979). Gelation of sickle cell hemoglobin in mixtures with normal adult and fetal hemoglobins. J Mol Biol 133(4): 435-467.

Verma IM (1990). Gene therapy. Sci Amer November: 68-84.

Visek WJ, Kolodny GM and Gross PR (1972). Ammonia effects in cultures of normal and transformed 3T3 cells. J Cell Physiol 80: $373-382$.

Address for correspondence: Bernhard O. Palsson, Department of BioEngineering, University of California-San Diego, 9500 Gilman Drive, La Jolla, CA 92093-0412, USA. 